\title{
Efectividad de la gestión administrativa en los gobiernos locales altoandinos, Luya, región Amazonas
}

\section{Effectiveness of administrative management in high Andean local governments, Luya, Amazonas región}

\author{
Keny Vásquez Fernández ${ }^{1}$ José Darwin Farje Escobedo ${ }^{2}$
}

\section{RESUMEN}

El presente trabajo de investigación tuvo como objetivo diagnosticar la efectividad de la gestión administrativa de los gobiernos locales altoandinos de Luya, región Amazonas; el estudio es descriptivo; la muestra estuvo conformada por 50 colaboradores que laboran en cinco municipalidades; el instrumento fue un cuestionario adaptado por el autor, de Ñañez (2017), y estuvo conformado por 30 preguntas en escala tipo Likert, con las categorías de eficiente, regular y deficiente. Los resultados obtenidos muestran la efectividad de la gestión administrativa en las municipalidades de la provincia de Luya; de las cinco municipalidades, para el 28,5\% de los encuestados, la gestión administrativa es eficiente; según el 49,0\%, la gestión es regular y el 22,5\% responde que la gestión administrativa es deficiente. En consecuencia, se concluye la efectividad en la gestión de los gobiernos locales estudiados, es predominantemente regular. Siendo la planificación una de las dimensiones que presenta mayor deficiencia en la gestión.

Palabras clave: Gestión administrativa, gobiernos locales

\begin{abstract}
TThe present research work aimed to diagnose the effectiveness of the administrative management of the high Andean local governments of Luya, Amazon region; the study is descriptive; the sample consisted of 50 collaborators who work in five municipalities; The instrument was a questionnaire adapted by the author, from Ñañez (2017), and consisted of 30 questions on a Li-kert-type scale, with the categories of efficient, regular and poor. The results obtained show the effectiveness of administrative management in the municipalities of the province of Luya; of the five municipalities, for $28.5 \%$ of those surveyed, administrative management is efficient; According to $49.0 \%$, management is regular and $22.5 \%$ respond that administrative management is deficient. Consequently, the effectiveness of the management of the local governments studied is concluded, it is predominantly regular. Planning being one of the dimensions that presents the greatest deficiency in management.
\end{abstract}

Keywords: Administrative management, local governments

\footnotetext{
Bachiller en Administración de Empresas egresado de la Facultad de Ciencias Económicas y Administrativas de la Universidad Nacional Toribio Rodríguez de Mendoza de Amazonas.

${ }^{2}$ Licenciado en Educación, docente de la Universidad Nacional Toribio Rodríguez de Mendoza de Amazonas, Doctor en Ciencias ambientales.
} 


\section{INTRODUCCIÓN}

La gestión administrativa cada vez tiene mayor importancia en la optimización de procesos que garanticen el funcionamiento organizacional, dado su carácter social, en cualquier escenario mundial. En nuestro país, las organizaciones del sector público, tienen dificultades en gestión, en los diferentes estamentos gubernamentales, según Chanamé (2017) las deficiencias de la gestión pública en el Perú, son: un sistema de planeamiento desconectado, los gobiernos no recogen las necesidades de la población; una estructura de organización y funciones ineficaces; una producción de bienes y servicios inadecuados; y finalmente una débil articulación gubernamental e interinstitucional.

Manuel Gonzáles Prada, hace un crudo diagnóstico de la realidad nacional, cuestionando la presencia de la corrupción de los políticos, la actitud de los abogados, que venden la justicia al mejor postor, la falsedad de los periodistas al dar la noticia; sintetizando una frase retórica en su libro Horas de Lucha: "El Perú es un organismo enfermo, donde se aplica el dedo brota el pus" (Quintanilla, 2018).

Quiroz (2013), en su obra Historia de la corrupción en el Perú, dice: "La falta de crecimiento se debe a la corrupción, más aún cuando los gobiernos han desperdiciado fondos públicos y privados, ahuyentaron las inversiones; criticando la actitud displicente y de resignación de los académicos que consideran la corrupción de las autoridades como hecho inevitable y que afecta al crecimiento; $y$, cuestiona la equívoca percepción popular que si acaso un político $<$ hace obras $>$ sus robos estarían perdonados".

Como se aprecia, la predominancia de la corrupción de autoridades y la limitada capacidad de gestión pública de gobernantes, hacen que estemos ubicados en el grupo de países "en vías de desarrollo"; al respecto, Ganoza y Stiglich (2015) refieren que "El crecimiento económico ha venido experimentando en los últimos años, no es una tendencia sostenible en el tiempo; desde el 2013, la economía se ha frenado, $\mathrm{y}$ es probable incluso, que entre en un franco proceso de retroceso". La realidad descrita ha motivado investigar sobre la efectividad de la gestión administrativa de los gobiernos locales altoandinos de la provincia de Luya.

Partiendo de esa realidad, se propuso como objetivo general, determinar la efectividad de la gestión administrativa de los gobiernos locales altoandinos de la provincia de Luya, durante el año 2019. En ese sentido, la investigación buscó, específicamente, diagnosticar la efectividad de la gestión administrativa en las dimensiones de planeación, organización, dirección y control, en los gobiernos locales altoandinos de la provincia de Luya.

Entre los estudios relacionados a la investigación, se consideran a Sosa (2015), al abordar Democracia, descentralización y cambio en las administraciones públicas de México (1982-2010), donde concluye, teniendo en cuenta que solamente el $25 \%$ hace la rendición de cuentas específicamente en los programas sociales; el Gobierno debe estar sometido al escrutinio público porque maneja fondos del estado, y el legislativo siempre debe cumplir la fiscalización y control político.

Por su parte, Ñañez (2017) en su estudio sobre gestión administrativa en las municipalidades de Azángaro y Chocos - Lima; concluye que en la planeación, no existe evidencias suficientes para afirmar diferencias significativas en ambas gestiones; mientras que en la organización, sí existen diferencias significativas en los resultados obtenidos; también existen diferencias significativas en la dirección, en la gestión de las dos municipalidades participantes del presente estudio.

Asimismo, Cabanillas (2015) en su estudio: propuesta de un plan de servicio al cliente (usuario), para mejorar la imagen de la municipalidad distrital de Sorochuco, en el período 2013-2014; concluyendo que la municipalidad de Sorochuco muestra debilidades en los servicios que brinda a la población, debido a que los trabajadores no reciben capacitación en temas de relaciones humanas y servicio al cliente, por tanto, brindan un mal servicio, generando incomodidad y desconfianza en los usuarios.

Calero (2016), concretó el estudio: gestión administrativas y calidad de los servicios en la municipalidad provincial de Huaral; concluye que la gestión administrativa debe ser conducida con personal calificado que brinde un buen servicio; donde haya una buena planificación, organización, liderazgo y por otro lado, un sistema eficiente de control; para el logro conjunto de objetivos institucionales.

Para abordar las variables de estudio, se consideran bases teóricas de diversos autores. Haciendo referencia a la administración, Chiavenato (2009) refiriéndose al enfoque neoclásico, refiere: "El enfoque neoclásico está asociado a la teoría clásica debidamente actualizada y redimensionada para enfrentar problemas administrativos vigentes en la organización". El enfoque es el que más relación tiene si se asocia a la función de los administradores, en ese contexto un administrador podría tomarlo como instrumento de gestión en cualquier empresa dada su condición de la administración como una disciplina antigua. Además, agrega, "Los neoclásicos se caracterizan por su heterogeneidad; esta teoría 
resalta aspectos prácticos de la administración, el pragmatismo y la búsqueda de resultados concretos y palpables; donde importa más el proceso; actualmente esta teoría se está imponiendo en la empresa pública y privad; considerando a la planeación, organización, dirección y control; como líneas eje".

Asimismo, de acuerdo a Chiavenato (2010) las funciones de los administradores son:

a) lograr que los recursos de la organización sean productos en términos económicos, minimizando riesgos y maximizando oportunidades; b) hacer que los recursos humanos se caractericen por su productividad. En consecuencia, es bueno ubicar a las personas a trabajar juntas y reunir en una tarea común sus habilidades y conocimientos, imponiendo sus fuerzas productivas sobre las debilidades; y, c) desempañar una función pública. El administrador es visible y representa algo en la comunidad (p. 370).

Las funciones de los administradores son fundamentales por lo que, deben direccionarse a tener un perfil con liderazgo, estar preparado para cualquier cambio dentro o fuera de la organización; además de efectuar sus funciones con eficacia, eficiencia, economía y calidad para que la misma sea exitosa.

Luna (2008) considera las siguientes características de la administración: i) Universalidad, la realidad de la administración se aplica en todo organismo social; nacionales o internacionales, públicas o privadas; ii) Especificidad o propósito, el conocimiento administrativo tiene un carácter específico, porque es un medio efectivo para lograr que se haga el trabajo, por lo consecuente no puede confundirse con otras disciplinas afines, iii) Unidad temporal; se integra mediante etapas, llamadas también fases que en el transcurso del tiempo se están aplicando sinérgicamente. El dinamismo de la administración abarca todas sus partes que la forman y éstas se dan como un proceso de mejora continua, iv) Unidad jerárquica o amplitud del ejercicio, la administración se ejerce en todos los niveles de gobierno social, vertical y horizontal, v) Interdisciplinaria, es semejante a todos aquellos conocimientos relacionados con eficacia y eficiencia; vi) Valor instrumental, constituye un medio orientado a alcanzar un fin; y vii) Flexibilidad, la administración tiene en cuenta algo de holgura para satisfacer expectativas sociales en la que opera.

Reyes (2009) refiriéndose a la unidad y división del proceso administrativo afirma que: "Todo proceso administrativo forma un continuo inseparable, en el que cada parte, cada acto, cada etapa, tiene que estar indisolublemente unida con las demás, y que, además operan simultáneamente".
Louffat (2012) sostiene que "la administración es el proceso encargado de administrar los recursos en las diversas áreas administrativas". Asimismo, permite accionar con sumo cuidado y criterio en el manejo de los recursos que cuenta la entidad o empresa; con el propósito de ser utilizados pertinentemente para alcanzar las metas trazadas.

Pérez (2004) al ocuparse de la "gestión" describió que: "está asociada a la idea de acción para que los objetivos fijados se cumplan. Los elementos necesarios para gestionar algo en resumen se basan al ciclo de la gestión".

En ese sentido, en una empresa pública o privada la gestión es un término clave puesto que se toma en cuenta un grupo de acciones y responsabilidades relacionadas a identificar programas, proyectos, actividades o tareas; luego medirlas minimizando riesgos y finalmente realizar un control adecuado para ver el cumplimiento de los objetivos iniciales.

En lo que respecta a las dimensiones de la administración, Chiavenato (2010) refiriéndose a:

La planeación afirma que: Las organizaciones no trabajan con base en la improvisación, casi todo lo que hacen es planeado con anticipación es la primera función administrativa, porque sienta las bases para las demás. Así mismo, es la que define cuáles serán los objetivos para alcanzar y que se debe hacer para llegar a ellos. Se trata de un modelo teórico para la acción futura. Comienza por establecer los objetivos y detallar los planes para alcanzarlos de la mejor manera posible. Planear es definir los objetivos y escoger de antemano el mejor curso de acción para alcanzarlos. La planeación define a dónde se quiere llegar, lo que se debe hacer, cuándo, cómo y en qué secuencia.

Por su parte Munch (2014) dice que la planeación, "es la determinación de los escenarios futuro y rumbo hacia donde se dirige la empresa, así como la definición de resultados y estrategias, buscando minimizando riesgos". En ese sentido, la planeación es proyectarse algo a futuro, con una dirección o fines claros y así poder lograr de manera positiva una producción o resultados, para ello la empresa o entidad debe considerar los riesgos ante cualquier circunstancia dentro proceso.

Mientras que, la dimensión de la organización, consiste en: a) Determinar las actividades específicas necesarias para alcanzar los objetivos planeados (especialización), b) Agrupar las actividades en una estructura lógica (departamentalización), c) Encargar las actividades a puestos y personas específicos (puestos y tareas)"; además agrega Munch (2014), que consiste en establecer de manera clara las 
acciones y funciones a realizar que fue determinado por la planeación, luego cada actividad será direccionada de acuerdo a lo que corresponda, la organización se debe determinar con efectividad los recursos físicos y humanos idóneos para lograr metas.

La dirección, en la idea de Chiavenato (2010, pág. 281), "implica mucha responsabilidad en la alta dirección de una organización; el directivo debe influir en los colaboradores para lograr los objetivos esperados; en consecuencia, el directivo debe caracterizarse por su liderazgo para una buena gestión”.

Finalmente, la dimensión de control, según Chiavenato (2010), "es asegurar que los resultados de lo planeado, organizado y dirigido se ciñan a objetivos establecidos previamente. La esencia del control reside en comprobar si la actividad que se controla alcanzará o no los objetivos o resultados deseados".

Haciendo referencia a la gestión pública, las organizaciones en la actualidad deben preocuparse por responder a los intereses generales de la sociedad, en ese sentido, el desempeño del Estado depende de la calidad y eficiencia de las políticas y el desempeño de la gestión pública. Para Milgrom y Roberts (2009), "es coordinar y motivar a las personas de una organización para conseguir unos objetivos".

Los términos "gestionar" y "administrar" aparecen como sinónimos, similares, de uso indistinto, incluso ambos son definidos como la acción y el efecto de hacer algo, asumir una función o tarea para cumplir un propósito. Sin embargo, en la legislación peruana estos conceptos aparecen diferenciados. Para Tello (2009), "la gestión pública se ocupa de la utilización de medios adecuados para alcanzar un fin colectivo, además, trata de los mecanismos de decisión para la asignación y distribución de recursos públicos, para lograr objetivos colectivos".

Al ocuparnos de los gobiernos locales, teniendo en cuenta el proceso histórico, el 16 de noviembre de 1853, el Congreso de la República del Perú, dio la primera Ley Orgánica de Municipalidades, como instancias promotoras del desarrollo local, con autonomía para gestionar intereses de la colectividad y administrar la prestación de servicios públicos locales y el desarrollo integral, sostenible y armónico.

Según la Ley $N^{\circ} 27972$, en su artículo $1^{\circ}$ establece:

Los gobiernos locales son entidades básicas de la organización territorial del Estado y canales inmediatos de participación vecinal en los asuntos públicos, que institucionalizan y gestionan con autonomía los intereses propios de las correspondientes colectividades; siendo elementos esenciales del gobierno local, el territorio, la población y la organización. Están conformadas por las municipalidades provinciales y distritales, tienen personaría jurídica y plena capacidad para el cumplimiento de sus fines. Presidencia del Consejo de Ministros" (2005). Según la Ley Orgánica de Municipalidades (LOM): El Consejo municipal, provincial y distrital, está conformado por alcalde y regidores que establezca el Jurado Nacional de Elecciones. Son atribuciones del alcalde: a) defender y cautelar los derechos e intereses de la municipalidad y los vecinos; c) ejecutar los acuerdos del concejo municipal; d) proponer al concejo municipal proyectos de ordenanzas y acuerdos; e) dirigir la formulación y someter a aprobación del concejo el plan integral de desarrollo sostenible local y el programa de inversiones concertado con la sociedad civil; entre otras.

\section{MATERIAL Y MÉTODO}

La presente investigación fue descriptiva de corte transversal, propositiva (Sánchez y Reyes, 2015). La muestra estuvo conformada por un total de 50 trabajadores que laboran en cinco municipalidades distritales, divididos en forma equitativa. El tamaño muestral fue determinado por el método no probabilístico, intencional o por conveniencia, en función al criterio de accesibilidad (Ary, 1987). Los métodos que se utilizaron fueron el inductivo, deductivo, analítico y sintético.

Se aplicó un cuestionario que permitió medir la gestión administrativa en los gobiernos locales, en las dimensiones de planeación, organización, dirección y control; lo conformaron 30 ítems, con preguntas de tipo Likert en función a las siguiente escala valorativa y numérica: siempre (3 puntos); A veces ( 2 puntos) y Nunca (1 punto). Para el procesamiento estadístico se establecieron las siguientes categorías: deficiente (1 30 puntos); regular ( $31-60$ puntos) y eficiente (61 90 puntos).

El proceso de adaptación del cuestionario, el análisis de confiabilidad y validez, se fundamentan en la Teoría clásica de los test Muñiz (2003). El cuestionario fue aplicado de manera anónima, se asignó un tiempo de 40 minutos por participante para responderla.

\section{RESULTADOS}

Tabla 1. Porcentaje de la gestión administrativa en la dimensión de planeación. 


\begin{tabular}{lcc}
\hline $\begin{array}{c}\text { Gestión administrativa } \\
\text { en planeación }\end{array}$ & No & \% \\
\hline Eficiente & 13 & 26.0 \\
Regular & 25 & 50.0 \\
Deficiente & 12 & 24.0 \\
Total & 50 & 100.0 \\
\hline
\end{tabular}

Tabla 2. Porcentaje de la gestión administrativa en la dimensión de organización

\begin{tabular}{lcc}
\hline $\begin{array}{c}\text { Gestión administrativa } \\
\text { en organización }\end{array}$ & $\mathbf{N}^{\circ}$ & $\mathbf{\%}$ \\
\hline Eficiente & 15 & 30.0 \\
Regular & 26 & 52.0 \\
Deficiente & 9 & 18.0 \\
Total & 50 & 100.0 \\
\hline
\end{tabular}

Tabla 3. Porcentaje de la gestión administrativa en la dimensión de dirección

\begin{tabular}{lcc}
\hline $\begin{array}{c}\text { Gestión administración } \\
\text { en dirección }\end{array}$ & $\mathbf{N}^{\circ}$ & $\mathbf{\%}$ \\
\hline Eficiente & 15 & 30.0 \\
Regular & 24 & 48.0 \\
Deficiente & 11 & 22.0 \\
Total & 50 & 100.0 \\
\hline
\end{tabular}

Tabla 4. Porcentaje de la gestión administrativa en la dimensión de control

\begin{tabular}{lcc}
\hline $\begin{array}{c}\text { Gestión administrativa } \\
\text { en control }\end{array}$ & No & \% \\
\hline Eficiente & 14 & 28.0 \\
Regular & 23 & 46.0 \\
Deficiente & 13 & 26.0 \\
Total & 50 & 100.0 \\
\hline
\end{tabular}

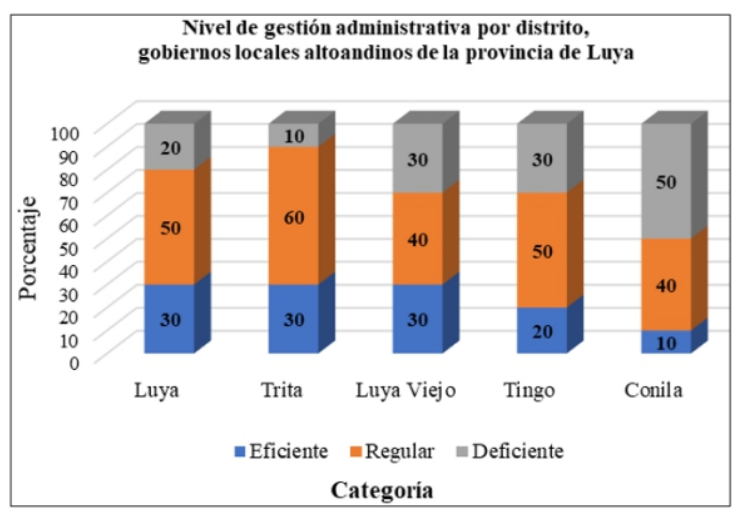

Figura.1. Porcentaje de nivel de gestión administrativa por distrito de los gobiernos locales de la provincia de Luya

\section{DISCUSIÓN}

Los resultados guardan relación con el diagnóstico de la Dirección Nacional de Presupuesto Público sobre la gestión municipal en el país, realizada por Torres (2007), "en cuanto al planeamiento, ni la tercera parte de las municipalidades cuenta con plan de desarrollo urbano y en la zona rural, la situación es más dramática, dado que solo un 3\% tiene planes de desarrollo". En el presente estudio, se aprecia que en la dimensión de planeación, el 24,0\%, está en el nivel deficiente y solo el $26.0 \%$, en el nivel eficiente; y, la mitad de los encuestados dice que la gestión es regular (tabla 1); encontrando similitudes con el estudio de Cabanillas (2015), con su investigación sobre la propuesta de un plan de servicio al cliente (usuario), para mejorar la imagen de la municipalidad de Sorochuco, concluye que la municipalidad muestra debilidades en los servicios a los usuarios, debido a que los trabajadores no reciben capacitación en temas de relaciones humanas y servicio al cliente, consecuentemente el servicio es malo, y los usuarios no confían y quedan insatisfechos con el servicio.

En la dimensión de organización en la prestación del servicio administrativo de las municipalidades altoandinas, el $18.0 \%$ dice que la organización, es deficiente, y el 52.0\%, refiere que es regular (tabla 2); hay coincidencias con el trabajo de Sosa (2015), en su trabajo sobre gestión pública en México, concluye que es importante la actuación del gobierno para verificar el ejercicio del gasto público, especialmente en materia de desarrollo social. Es decir, asociándolo a nuestra realidad, tiene que haber fiscalización para garantizar que los presupuestos sean invertidos en los programas para los que fueron dados para no caer en posibles faltas de malversación.

Lo mismo ocurre con la efectividad de la gestión en las dimensiones de dirección y control, dado que, el $22.0 \%$ refiere que el servicio municipal es deficiente; y, para casi la mitad $(48,0 \%)$, es regular; en el segundo caso (control), el $26.0 \%$ sostiene que el nivel es deficiente y para el $46 \%$, es regular; lo que significa que la gestión en la dirección y control, no son eficientes (tablas 3 y 4). Encontrando similitudes con la investigación de Nañez (2017), sobre la gestión administrativa en las municipalidades de Azángaro y Chocos, Lima; donde concluye que existen diferencias significativas en la dirección en ambas municipalidades; debido a aspectos relacionados con el perfil profesional de los colaboradores que obstaculiza para cumplir funciones con eficiencia. Cuando se hace la comparación de la efectividad de la gestión administrativa por gobierno altoandino, se aprecia que hay un predominio de los niveles deficiente y regular en los cinco distritos, debido a la limitada cualificación de los servidores públicos (figura 1); lo que significa que hay semejanzas con el estudio de Calero (2016), concluye que la gestión 
debe ser conducida con personal calificado que brinde un buen servicio, donde haya una buena planificación, organización, liderazgo y por otro lado, un sistema eficiente de control; para el logro conjunto de objetivos institucionales.

\section{CONCLUSIONES}

El nivel de gestión administrativa en la dimensión de planeación es deficiente con un $24 \%$, regular con un $50 \%$ y es eficiente según el $26 \%$.

El nivel de gestión administrativa en la dimensión de organización es deficiente para el $24 \%$ de trabajadores, es regular con un $52 \%$ y es eficiente para el $30 \%$.

La efectividad de la gestión administrativa en la dimensión de dirección es deficiente para el $22 \%$, regular para el $48 \%$ y es eficiente según el $30 \%$.

La efectividad de la gestión administrativa en la dimensión de control es predominantemente regular para el $46 \%$, y deficiente para el $26 \%$ de los trabajadores.

En la efectividad de la gestión administrativa, por gobierno altoandino distrital, hay han un predominio del nivel regular con rangos que van desde el $40 \%$ al $60 \%$ en las cinco municipalidades.

La municipalidad que presenta mayor dificultad en su gestión es Conila, con el $50 \%$ en el nivel deficiente.

\section{REFERENCIAS BIBLIOGRÁFICAS}

Ary, D. (1987). Introducción a la investigación pedagógica. Interamericana S.A.

Cabanillas, L. (2015). Propuesta de un plan de calidad de servicio al cliente (usuario), para mejorar la imagen de la Municipalidad distrital de Sorochuco, período 2013-2014. (Tesis de pregrado). Universidad Nacional de Cajamarca.

Calero, J. (2016). Gestión administrativa y calidad de los servicios en la Municipalidad provincial de Huaral en el año 2015, (tesis de pregrado). Un i vers i d a d o s Ángele s. http://repositorio.uladech.edu.pe/bitstream/ha ndle/123456789/573/CALERO_MORA_JANET H_DEL_PILAR_GESTION_CALIDAD_HUARA
L.pdf?sequence $=1$

Chanamé, C. (2017). Deficiencias de la gestión pública en Perú que se deben resolver. Lima, Perú: Universidad Continental.

Chiavenato, I. (2009). Administración de Recursos Humanos. McGraw Hill.

Chiavenato, I. (2010). Comportamiento Organizacional. La Dinámica del éxito en las organizaciones. McGrawHill.

Chiavenato, I. (2011). Administración de recursos humanos. El capital humano de las organizaciones. (8a.ed). McGrawHill.

Ganoza, D. y Stiglich, A. (2015). El Perú está calato. Planeta.

Louffat, E. (2012). Administración: Fundamentos del proceso administrativo. (3a ed.). Editorial Cengage Learning.

Luna, A. (2008). Proceso Administrativo. Patria.

Milgrom, P. y Roberts, J. (2009). Economics, organization and Management. EUA. PrenticeHall.

Munch, L. (2014). Administración: Gestión organizacional, enfoques y proceso administrativo. ( $2^{\mathrm{a}} \mathrm{ed}$.). Editorial Mexicana.

Muñiz José. (2017). Teoría Clásica de los Test. (2 ${ }^{\mathrm{a}}$ ed.). Pirámide.

Ñañez, E. (2017). Gestión administrativa en las municipalidades de Azángaro y Chocos - Lima, 2016, (tesis de posgrado). Universidad Cesar Vallejo. Trujillo, Perú.

Pérez, J. (2004). Gestión por procesos. (3 ${ }^{\mathrm{a}}$ ed.). ESIC Editorial.

Presidencia del Consejo de Ministros (2005). Ley Orgánica de Municipalidades. Lima, Perú.

Quintanilla, A. (12 de agosto de 2018). Donde se pone el dedo, salta la pus. Correo. https://diariocorreo.pe/opinion/donde-sepone-el-dedo-salta-la-pus-830736/?ref=dcr

Quiroz, A. (2013). Historia de la corrupción en el 
Perú. Instituto de Estudios Peruanos

Reyes, A. (2009). Administración moderna. Limusa.

Sánchez, H. y Reyes, C. (2015). Metodología y diseños en la investigación científica. (5a. ed). Business SupportAneth.

Sosa, J. (2015). Democracia, descentralización y cambio en las administraciones públicas de México (1982-2010), (tesis de posgrado). Universidad Complutense de Madrid, España.

Tello, P. (2009). Gestión Pública. Programa de formación: desarrollo de capacidades para el fortalecimiento de las organizaciones políticas. Programa de Naciones Unidas para el Desarrollo.

Torres, S. (2007). Diagnóstico de la gestión municipal. Dirección Nacional del Presupuesto Público del Ministerio de Economía y Finanzas. Lima, Perú. 